\section{A (tiniral}

\author{
ON
}

\section{MUSCLE-TONUS, TONIC RIGIDITY, AND TONIC FITS. \\ BY}

SiR JAMES PURVES STEWART, K.C.M.G., C.B., SENIOR PHYSICIAN TO THE WESTMINSTER HOSPITAL.

Ordinarily our voluntary muscles, even at rest, are in a state of slight tonus, a sort of braced-up condition. This muscle-tonus is a reflex affair-that is, it is dependent, primarily, upon the integrity of the spinal reflex arc. The reflex arc, as we all know, consists of an afferent limb, made up of (1) the sensory nerve, leading up to the posterior spinal root, then (2) a short intermediate neurone within the grey matter of the spinal cord, and, lastly, (3) an efferent limb, consist. ing, in series, of the anterior cornual cell, the anterior norve root, the motor nerre and the muscle.

\section{Diminished Muscle-Tonus:} Hypotonia.

A lesion interrupting any part of this spinal reflex arc will cause, among other phenomena, loss of muscle. tonus. If the lesion be in the sensory or afferent limb -for example, in the pos. terior spinal root-it is often associated with sensory loss in the corresponding root. area; if it is the efferent or motor limb which is interrupted, it is associated with muscular weakness and atrophy. A good example of loss of muscle-tonus from lesion of the posterior roots is the muscular hypotonia of tabes, which is sometimes so extreme that the patient's limbs can be placed in all sorts of unusual postures. An illustration of loss of muscle-tonus from a lesion of the motor part of the reflex arc is seen in paralysis of any motor nerve-for example, the posterior inter.

osseous branch of the musculo-spiral, with its charac. teristic flaccid drop.wrist.

Increased Muscle-Tonus: Rigidity.

The opposite condition of excessive reflex activity with increased muscle-tonus may arise from irritative conditions in the reflex arc. Sometimes the irritation is in the afferent limb of the arc, as can be easily observed in the localized rigidity of the muscles around a painful or inflamed joint or the localized abdominal rigidity of an inflamed appendix. It may also result from irritative conditions of the anterior cornual cells-for example, in poisoning by strychnine or by the toxin of tetanus, although in tetanus, as we shall see later, other factors are probably concerned.

\section{Pyramidal and Extra-Pyramidal Tracts Influencing Muscle.Tonus.}

All the movements of our voluntary muscles are executed through the anterior cornual cells of the spinal cord. These receive two different varieties of motor impulses from the brain. The first class of impulses are voluntary motor impulses, from the pre-Rolandic cortex, travelling down the brain-stem along the pyramidal tracts. The second class are involuntary non-pyramidal impulses, from subcortical motor centres (such as the corpora striata, the red nuclei, the accessory vestibular nuclei of Deiters, etc.) travelling along subcortico-spinal tracts (such as the rubro-spinal in the lateral columns, the restibulo-spinal in the anterior columns, etc.). The accompanying diagram, by Professor Stopford, of Manchester, shows what I mean. The subcortical ganglia are again influenced or reinforced by the intracerebellar nuclei (constituting cerebello. rubro-spinal and cerebello-vestibulo-spinal paths, and so on). These two sets of impulses, cortical and subcortical, pyramidal and extra-pyramidal, acting on the motor cells of the spinal cord, influence the motor activity of the anterior cornual cells, the pyramidal impulses producing voluntary movements, the non-pyramidal impulses producing automatic acts. But, in addition, they have a regulating effect upon muscle-tonus and posture which is mutually antagonistic, so that, if both sets of impulses be knocked out, the normal muscular tonus is diminished and the affected muscles become not only paralysed, but also flaccid. If, howerer, either the pyramidal impulses, on the one hand, or the subcortical impulses, on the other, are kuocked out alone, the other partner is left unopposed, and the affected muscles become hypertonic and rigid, producing so-called spasticity.

The spasticity due to pure pyramidal disease-for example, in ordinary hemi. plegia-is produced by the un opposed non-pyramidal motor tracts. These act on the anterior cornual cells, and produce hypertonus in the affected muscles, which, although paralysed for voluntary movements, nevertheless can still be influenced by the subcortical centres in the corpus striatum, red nucleus, etc., so that certain automatic movements can be performed in the paralysed limb or limbs.

On the other hand, in the spasticity due to pure extra-pyramidal clisease (for example, in paralysis agitans, in progressive lenticular degeneration, etc.) automatic movements, such as those of swinging the arm in walking, blinking the eyelids, certain emotional movements of the face, etc., are dimin. ished or lost, and yet the patient, owing to integrity of his pyramidal tracts, can still perform all ordinary voluntary movements with his spastic muscles.

When both sets of motor impulses, pyramidal and nonpyramidal, are cut off, as in a total transverse lesion of the spinal cord (say from a fracture-dislocation or from a bullet wound), the muscles below the level of the lesion at once become paralysed and we have a flaccid paraplegia-that is, one in which the paralysed muscles are devoid of muscle-tonus. This flaccid stage lasts from one to three weeks. If, howerer, the spinal cord lesion is incomplete, so that some fibres, whether pyramidal or non-pyramidal, still reach down to the anterior cornual cells below the level of the lesion, we then find that muscle tonus is preserved and increased from the start-that is, that the paraplegia is spastic in type. The transient loss of deep reflexes in a total transverse lesion compared with their exaggeration from the very start in an incomplete transrerse lesion, is another point of interest, but we shall not discuss it on this particular occasion.

\section{Decerebrate Rigidity.}

Let us now consider what happens with a still higher transverse lesion of the brain-stem, away above the spinal cord and medulla altogether, at a level which cuts off the cerebral hemispheres, together with their voluntary motor impulses, but leaves intact the great subcortical mechanisms of the red nuclei and vestibular nuclei, also leaving these ganglia free to be influenced by tine undamaged cerebellum. When this is done experimentally 
in animals, by transection through the brain-stem in the mesencephalon, the result is to produce so-called " decerebrate rigidity" in which the animal's limbs, both upper and lower, are rigidly extended, whilst the neck and whole spinal column, right down to the tail, are tonically hyperextended with retraction of the head. We note particularly that this lesion is a purely destructive or "negative" lesion.

In. rare cases in human patients we may observe a fairly complete reproduction of acute decerebrate rigidity from unopposed activity of the subcortical motor centres. The following is an example:

In November, 1903, F. G., a man of 40, with albuminuria and arterio-sclerosis, had an attack of sudden right-sided hemiplegia of the face, arm and leg, with flaccidity of the paralysed limbs and unconsciousness. After an hour he recovered conscionsness and was able to recognize his surroundings and to answer "Yes" and "No" to questions.

Three hours later he again suddenly became comatose with an entirely different group of symptoms. On top of the pre existing flaccid right hemiplegia there appeared strong bilateral tonic spasm of the trunk, arms, and legs. The upper limbs were rigidly adducted at the shoulders, extended at the elbows, and hyperpronated at the forearms, so that the backs of the hands faced each other. The thumbs were turned invards within the loosely flexed fingers. The lower limbs were rigidly extended at hips, knees and ankles, whilst there were occasional sharp sudden symmetrical dorsiflexion movements of both ankles. The spinal muscles were also rigid. The fingers and toes were flaccid. The upper limbs occasionally became flaccid for a few minutes, but the lower limbs remained rigid to the end. 'l'he pupils were small and inactive to light. The breathing was stertorous, with flapping of the right cheek. The kneejerks were exaggerated and there was bilateral ankle clonus. The plantar reflexes could not be elicited owing to the frequent tonic spasms of the ankles.

The rigidity of the lower limbs and trunk persisted until death, which occurred four and a half hours from the onset of the original hemiplegic attack. The temperature ran up to $102^{\circ}$ at the end. The pupils became widely dilated, the left being larser than the right; both were oval in outline, the left with its long axis horizontal, the right with long axis almost vertical. The pulse was 186 at the end.

At the autopsy the left lateral ventricle was distended with a large blood clot, and the left corpus striatum was ploughed up by haemorrhage. The other ventricles of the biain were normal.

Decerebrate rigidity in man is the result of a destructive lesion, usually a haemorrhage, in the mid-brain. This haemorthage often spreads at the same time into the cerebral ventricles. It has been suggested that it is the intraventricular site of the haemorrhage which is the cause of the decerebrate rigidity, but I have repeatedly observed cases of extensive intraventricular haemorrhage in which there has been no rigidity whatever. It therefore seems to me probable that some other factor, such as oedema of the brain-stem, must be the decisive one in cutting off the cerebral pyramidal impulses and leaving the subcortical centres uncontrolled.

Acute decerebrate rigidity is usually a terminal pheno. menon in a dying patient; but not always, as the following example will show:

Only last week I saw an old gentleman of 85 who had had $t$ wo attacks of left-sided hemiplegia-the first at the age of 73 , the second at 83-apparently due to cerebral thrombosis. Two months ago he had a couple of attacks of sudden unconsciousness and stertor, with half an hour's interval between them. In both of these he showed typical decercbrate rigidity, with In both of these he showed typical decercbrate rigidity, with limbs being tonically extended at the elbows and wrists, the metacarpo-phalangeal joints rigidly semi-flexed and the interplialangeal joints rigidly extended. The coma lasted forty minutes in the first attack and twenty minutes in the second. The pulse ran up to 120 during each attack. Throughout the attack he made champing movements of the jairs. For a few minutes after recovering consciousness he was mentally confused, but soon became bright and alert again, with no evidence of aggravation of his previous slight hemiplegia. He then went of aggravation of his previous slight hemiplegia. He then went couple, of similar character but milder in degree. From these again he has made an excellent recovery.

\section{Incomplete Deccrebrate Rigidity.}

Short of complete decerebrate rigidity, we sometimes meet with phenomena of chronic incomplete decerebrate rigidity-for example, in cases of cerebral diplegia, and. to a less extent, in ordinary hemiplegia.

In a decerebrate animal with tonically extended limbs, if we turn its head passively to one side or to the other, there occurs, after a short interval, a curious exaggeration of extension in the upper limb towards which the animal's face is turned (so-called facial limb), together with a diminution of the extension of the upper limb towards which its occiput is turned (occipital limb). Similarly, in certain cases of cerebral diplegia in which the subcortical centres are released from control, we can elicit so-called "conjugate automatic movements," so that, if the patient's head be turned, say, to the right, we observe that the left upper limb slowly becomes tonically flexed, whilst the right becomes extended. This phenomenon takes about a minute to reach its maximum. If we now turn the head passively to the opposite side, the attitude of the upper limbs becomes reversed.

\section{Rigidity in Pyramidal Lesions.}

Similar phenomena of decerebrate rigidity can some. times be demonstrated in chronic hemiplegia, being limited, of course, to the hemiplegic limbs. Thus we may observe involuntary flexion of the hemiplegic upper limb when the face is turned away from the hemiplegic side, and involuntary extension of the hemiplegic limb when the face is turned towards the hemiplegic side.

Many healthy people make automatic extension movements of the upper limbs during the act of yawning (which of course is a subcortical plienomenon). By the ancients such movements were termed "pandiculation." These movements often remain well marked in the paralysed limbs in a case of severe chronic hemiplegia when the patient yawns or is tickled. The transient tonic extension which then occurs in the previously flexed rigid fingers, or the elevation of the paralysed arm, may delude the patient with the vain hope that be is beginning to recover voluntary power. But, unfortunately, these movements are not a hopeful sign. On the contrary, being due to uncontrolled activity of the subcortico-spinal motor tracts, the more profound the lesion of the pyramidal tract has been, the more marked is the pandiculation likely to be.

The well-known muscular rigidity with which we are all familiar as forming part of the classical syndrome of a pyramidal or upper motor neurone lesion, is easily recog. nised as due to uncontrolled activity of the subcortico. spinal motor tracts. In such a lesion the posture of the spastic upper limb in cerebral hemiplegia or monoplegia tends to be one of flexion of the elbow with pronation of the forearm and flexion of the wrist and fingers. The posture of the spastic lower limb is usually one of extension of the hip and linee, with plantar flexion and inversion of the ankle and slight dorsiflexion of the toes. These postures of the limbs occur in pure pyramidal lesions-for example, in ordinary hemiplegia of cerebral origin or in pure lateral sclerosis of the spinal cord with the "extended" type of paraplegia.

Rigidity in Extra-Pyramidal Lesions.

Muscular rigidity also occurs in pure extra-pyramidal disease from unopposed action of the pyramidal tracts. This is typically seen in paralysis agitans, in which rigidity of the face, trunk and limbs (generally beginning unilaterally) invariably accompanies the tremor and usually precedes it. In fact, severe rigidity may be present for montlis before tremor supervenes, or tremor may even remain absent, when we have the so-called "paralysis agitans sine agitatione."

On the other hand, if the pyramidal and the extra. pyramidal tracts are both affected together, as for example in a diffuse spinal cord lesion, we no longer find the decerebrate extended posture of the lower limbs; on the contrary, owing to loss of tonus in the paralysed extensor muscles, there is flexion of the hip and knee with dorsiflexion of the ankle, so that the limb as a whole is drawu up or shortencd-the so-called flexed type of paraplegia.

\section{Cerebellum and Muscle-Tonus.}

Let us now turn to the cercbellum and its influence upon muscle-tonus. The fundamental function of the cerebellum is that of muscular synergia or co-ordination. For the efficient performance of co-ordinated muscular acts a certain degree of muscle-tonus is necessary. The cerebellar cortex is mainly a receiving platform for afferent, cerebello-petal impulses, for example, those of equilibration from the semicircular canals, also the proprioceptive impulses from muscles and joints, impulses which normally do not rise to consciousuess, although when they are disordered acute discomfort is at once felt. The motor 
centres of the cerebellum are located mainly in the intra. cerebellar nuclei, including the dentate, roof, and emboliform nuclei. These nuclei exercise their influence upon the anterior cornua and the voluntary muscles, not directly, since there are no direct, efferent, cerebello-spinal paths leading down to the spinal cord. The action of the cerebellum on the brain-stem is an indirect one, thrown in, as it were, from a projecting branch. It is exercised mainly upon the red naclei and upon the accessory vestibular nuclei of Deiters, from which, as we have seen (see figure), descending rubro-spinal and vestibulo-spinal tracts lead down into the main stem of the spinal cord to influence the activity of the anterior cornual cells. Each half of the cerebellum is connected with the contralateral red nucleus and with the ipsilateral nucleus of Deiters - that is, with the ipsilateral side of the spinal cord, since the rubrospinal tract decussates before reaching the spinal cord, whilst the vestibulo-spinal tract runs down uncrossed in the ipsilateral side of the cord.

\section{Destructive or Negative Cerebellar Lesions.}

If the cerebellum be experimentally destroyed the tonus of the voluntary muscles is at once diminished. In unilateral cerebellar lesions this loss of tonus is confined to the ipsilateral limbs and trunk. The affection of the trunk muscles in unilateral lesions of the cerebellum is dramatically shown by the fact that the tonic action of the contralateral muscles being now unopposed, the animal rotates around its own long axis in a. screw-like fashion. In a case of destruction of the right half of the cerebcllum this rotation is in the direction of screwing in a screw ; in left-sided lesions the direction is reversed (the animal's head representing the head of the screw).

Destruction of the anterior part of the middle lobe, or vermis, of the cerebellum affects the tonus of spinal muscles on both sides, so that in destruction of the front part of the vermis the animal falls forwards, whilst if the posterior part of the vermis be removed the animal falls backwards.

In human patients sudden destructive or negative lesions of the cerebellum are comparatively uncommon, but typical examples are occasionally met with in war. In unilateral wounds of the cerebellum the ataxia of the limbs is on the same side as the lesion. It is accompanied by hypotonia of the muscles on the affected side, so that the lìnb "flops about" and can be placed passively in abnormal postures. 'This hypotonia, however, tends to pass off after a week or two. In bilateral wounds of the cerebellum not only the limbs but the spinal and neck muscles become specially hypotonic, so that the patient cannot stand or even sit up unless he is supported. After a short time, if the lesion be stationary, compensation occurs, and the hypotonia rapidly subsides.

\section{Irritative or Positive Cerebellar Lesions.}

Lastly, we have to consider the effects of irritative or "positive" lesions of the cerebellum. These will increase muscular tonus and produce tonic rigidity of the affected parts. The mechanism through which this is effected is probably by means of intra-cerebellar nuclei, not the cerebellar cortex. Stimulation of the upper part of the dentate nucleus has been shown by Horsley and Clarke to produce deviation of the head and eyes towards the ipsilateral side. Stimulation of the basal portion of the dentate nucleus, together with the upper portion of the other adjacent intra-cerebellar nuclei on the same side, produces tonic flexion of the ipsilateral elbow, whilst stimulation of the paracerebellar nuclei alone, without the dentate uncleus, produces tonic extension of both legs, closely rescmbling the posture of "decerebrate rigidity."

Tonic or Cerebellar Fits.

In rare cases we may come across rigidity in man from irritative cerebellar lesions, producing a variety of "tonic fits." In these so-called cerebellar fits the posture of the lower limbs in extension is what we have already described, but that of the upper limbs is not quite the same as in ordinary decerebrate rigidity from a negative or destructive lesion of the brain-stem above the red nuclei. In irritative cerebellar lesions the typical hyperpronation of the forearms is often absent.

Let me relate to you an example of these "cerebellar fits" which some of you have witnessed in the wards.
R. N., a little boy of 4 years, was healthy until the age of 39. when he began to have "fits." At first about two nsed to occur in every twenty-four hours, one during sleep and another on waking in the morning, but after a couple of months they rapiderea in frequency, and by the time he came under observation they were occurring about every half-hour or so, day and night. Together with the increase in the fits, the child's intelligence rapidly deteriorated. Previously bright, active and lively, he became dul, apathetic, and neglectful of
the sphincters; his speech, previously fluent, became less distinct, so that he intermingled inarticulate noises with his almost monosyllabic, indistinctly articulated words. He occasionally placed his right hand on the side of his head, as if in pain. There was no vomiting. A week before I first saw him he became unsteady in his gait and occasionally fell when walking.

When first examined, on May 1st, 1920, he was mentally dull, but he understood and executed verbal requests to shut his eyes, put out his tongue, etc. He named accurately objects shown to him, such as a watch, penny, and scissors, but articulated his words very indistunctly. Tíhe optic discs and fundi were normal. The pupils were equal and normal in reaction. There was a fine rotatory nystagmus, only noticeable during ophthalmoscopy. The cranial nerves were otherwise normal. There was no cutaneous anaesthesia or analgesia, and all the limbs were powerful. The gait was extremely unsteady and reeling, so that he freguently fell, but not constantly in any special direction. The knee-jerks were brisk and equal; the ankle-jerks were absent. The plantar reflexes were flexor in type, and the abdominal reflexes were brisk. Kernig's sign was negative.

During examination he had one of his fits, of which the following is a detailed description. The child suddeuly became momentarily unconscious with cyanosis of the lips and face, dilatation of the pupils and turning of the eves slightly to the right side, with transient flickering of the eyelids. Meanwhile all his limbs became rigid. The upper limbs became tonically extended at the elbows and rigidly flexed at the wrists and metacarpo-phalangeal joints; the lower limbs were rigidly extended at the hips, knees and ankles, and inverted at the ankles. After lasting about twenty seconds the elbows became semiflexed and the fingers tritched slightly for about ten seconds, the lower limbs remaining rigidly extended as before. seconds, the lower limbs remaining rigidly extended as before. There was no biting of the tongue nor affection of sphincters during the attack.

The patient was kept under observation in Nestminster Hospital for two months, during which he had numerous daily attacks similar to the above, numbering from seven to twelve in the dartime and four to six at nights during sleep. At the onset of the fits the eves were turned sometimes to the right side, sometimes to the left. After an attack the child was not stuporous but seomed wide-awake, and went on plaving with his toys.

Radiograms of the skull showed no abnormality, and the optic discs remained normal. Bromide medication bad no apparent effect on the frequency of the fits.

In view of the persistence of these cerebellar attacks I asked my colleague, Mr. Spencer, to do a decompressive operation. Accordingly, on July 23 rd and 27 th a two-stage operation was Accried out in which both cerebellar hemispheres were widely exposed, the straight sinus between them being divided and the dura freely opened. The cerebellum bulged markedly, especially its right hemisphere, but no tumour was seen or felt in any accessible part of the cerebellum. The wound was closed and healed uneventfully, but the flap remained bulging,

During the early half of August, following the operation, the fits continued for a time, but with much diminished frequency. In the latter half of Ausust only four attacks occurred, and from August 30th onwards they ceased altogether.

When re-examined on October 26th, 1920, and again on January 4th, 1921, he was bright and intelligent, with normal speech and articulation. The optic discs and cranial nerves were normal. He was free from nrstagmus. There was no weakness or ataxia of the limbs, and he walked and ran about like other children, but was said to be somewhat excitable and impetuous in temper. The operation flap bulged as before.

I may also remind you that many years ago Hughlings Jackson ${ }^{1}$ regarded the phenomena of tetanic convulsions, with their tonic spasm of the face (risus sardonicus), jaws, neck, trunk and limbs, as a variety of cerebellar fit. It is probable, howerer, that in tetanus the mid-brain nuclei and the anterior cornual cells are also implicated in the irritative process.

Another point of interest is raised by the head retraction and spinal rigidity of basal meningitis. Whether this is also to be regarded as a variety of cerebellar irritation is well worth consideration.

Time doas not permit of our attempting any adequate discussion of other incomplete varieties of tonic fits. But those of you who are interested in the matter will find many suggestive points in a recent paper by Dr. Kinnier Wilson," where the subject is discussed in greater detail. 